\title{
The physiology of ketosis and the ketogenic diet
}

\author{
T Leonard iD
}

Department of Anaesthesia, School of Clinical Medicine, Faculty of Health Sciences, Chris Hani Baragwanath Academic Hospital, University of the Witwatersrand, South Africa

Corresponding author, email: tristan.leonard@wits.ac.za

\section{Summary}

Ketones are an important fuel source for the body during periods of starvation and are readily used by the brain, the heart, and skeletal muscle. Ketones may also be produced in response to certain diets. Interest in the use of a diet high in fat and low in carbohydrates in order to induce a state of ketosis has increased in recent years. This review will cover the physiology of ketosis and examine the effects of the ketogenic diet.

Keywords: ketones, ketosis, ketogenic diet, nutrition

\section{Introduction}

Ketone bodies are formed in the liver from fatty acid oxidation and in humans consist of three molecules, acetoacetate (AcAc), 3 - $\beta$-hydroxybutyrate (3HB) and acetone. ${ }^{1,2}$ AcAc is produced when carbohydrate stores are low and fatty acids are metabolised to produce acetyl CoA, which overwhelms the hepatocyte mitochondrial capacity to remove acetyl CoA. ${ }^{1}$ Two acetyl CoA molecules combine to form AcAc, which can be reduced with $\mathrm{NADH}$ to form $3 \mathrm{HB} .^{1}$ Acetone is formed from the spontaneous degeneration of AcAc. ${ }^{2}$ The liver is the only organ that produces ketone bodies but it cannot utilise ketones itself. ${ }^{1}$

\section{Ketogenesis}

Ketogenesis occurs in the liver during periods of low carbohydrate availability and ketones are an important fuel source for the brain and cardiac and skeletal muscle during starvation. Ketones also reduce the amount of glucose and protein utilised for energy production when glucose levels are deficient. ${ }^{2}$ When carbohydrate and glucose levels are low, fatty acid production is stimulated by the release of adrenaline and glucagon. Acetyl CoA is produced from $\beta$-oxidation of fatty acids and can enter the citric acid cycle. However, acetyl CoA has to combine with oxaloacetate in order to be able to enter the citric acid cycle. Oxaloacetate is produced from pyruvate during glycolysis and hence if glucose levels are low there is not enough oxaloacetate production and the remaining oxaloacetate is preferentially used for gluconeogenesis (Figure 2). ${ }^{2}$ The abundance of acetyl CoA that then remains forms ketone bodies (Figure 3 ). ${ }^{2}$
FAT LIVER

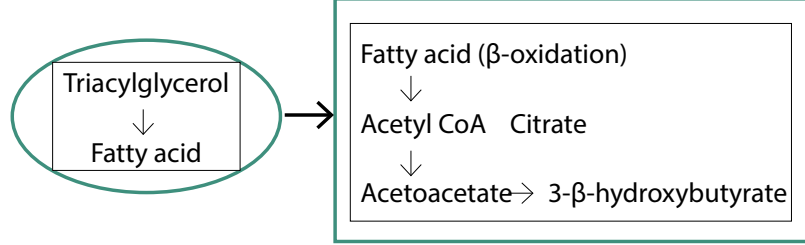

Figure 1: Ketone acid synthesis ${ }^{1}$

These ketone bodies can freely diffuse across cell membranes and can be used easily by the brain (and other tissues) as an energy source. ${ }^{3}$

Ketogenesis is controlled by three enzymes: hormone-sensitive lipase (in adipocytes), acetyl CoA carboxylase and HMG CoA synthase (in the liver). Hormone-sensitive lipase and acetyl CoA

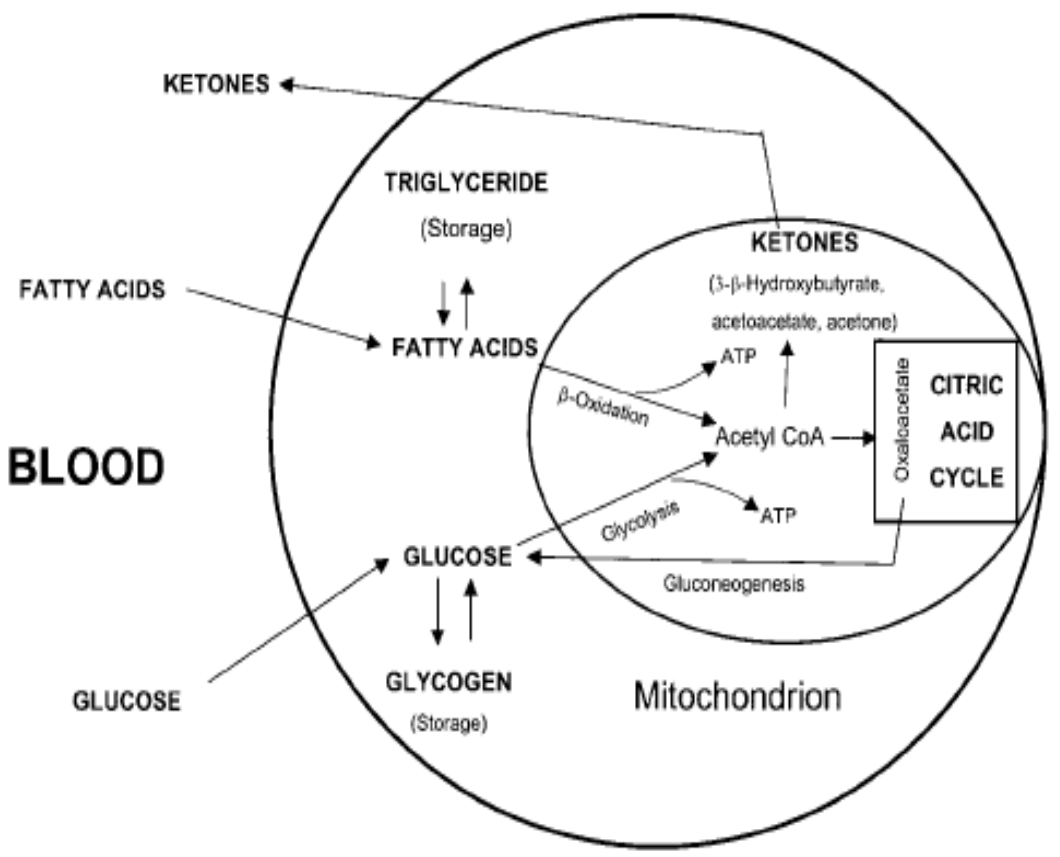

Figure 2: Relationship between glucose and fatty acid metabolism² 


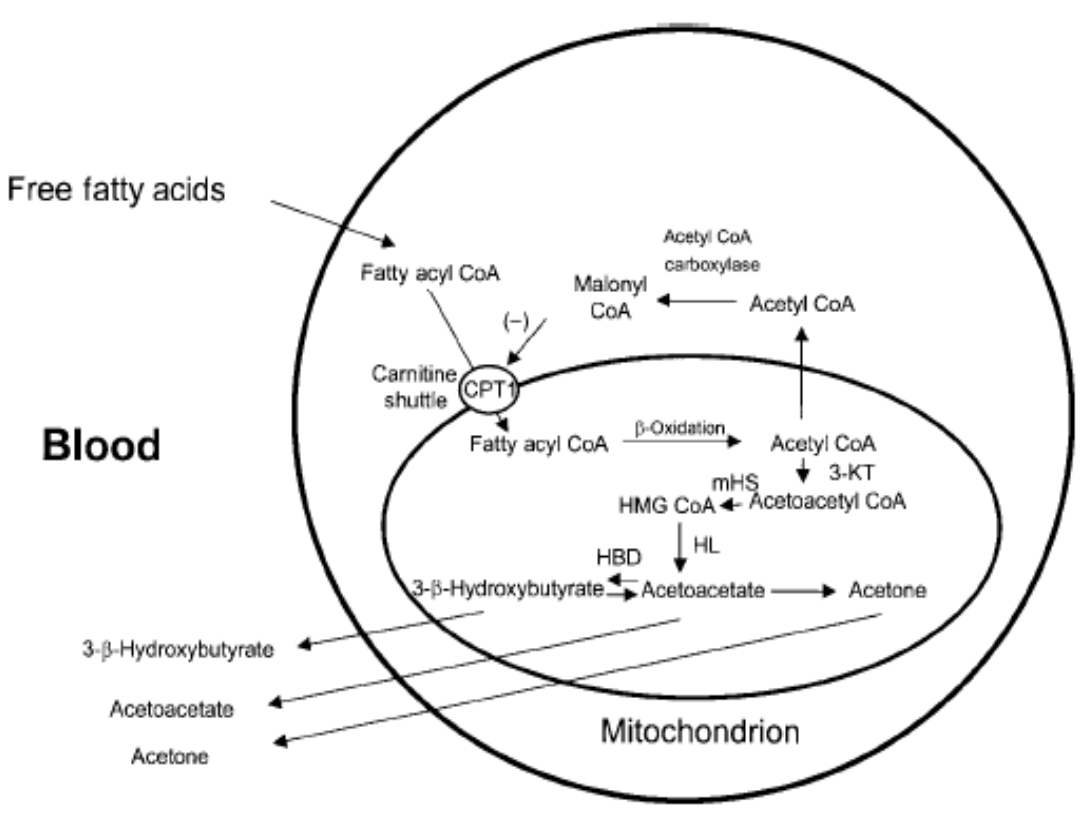

Figure 3: Enzymes involved in ketone body formation ${ }^{2}$

carboxylase are controlled by insulin ${ }^{4}$ (which inhibits ketogenesis) and adrenaline and glucagon ${ }^{5}$ (which stimulate ketogenesis). Hormone-sensitive lipase is stimulated by glucagon release (and inhibited by insulin) and breaks down triglycerides into fatty acids, resulting in raised serum fatty acid levels which leads to the production of increased amounts of acetyl CoA, the substrate for ketone synthesis. ${ }^{2}$ Conversely acetyl CoA carboxylase is stimulated by insulin (and inhibited by glucagon) and acts in the liver to convert acetyl CoA to malonyl CoA which blocks the transport of fatty acids into the mitochondria and reduces ketogenesis. Low levels of malonyl CoA will stimulate ketogenesis. HMG CoA synthase is in the hepatic mitochondria and converts acetoacetyl CoA into acetoacetate. This is the rate limiting step in producing ketone bodies and is stimulated by glucagon (and inhibited by insulin). Therefore, insulin inhibits ketogenesis by inhibiting hormone-sensitive lipase and stimulating acetyl CoA carboxylase, and glucagon (and adrenaline) drives ketogenesis through the activation of hormone-sensitive lipase and HMG CoA synthase. ${ }^{2}$

\section{Ketolysis}

Ketolysis is the process whereby ketone bodies are broken down in order to provide energy to tissues. It occurs in the mitochondria of many organs (except the liver which cannot utilise ketone bodies). There are two enzymes involved in ketolysis, succinyl CoA-oxoacid transferase (SCOT) and methylacetoacetyl CoA thiolase (MAT). Ketones enter the mitochondria from the blood via the monocarboxylate transporter 1 (MCT1) 6 and AcAc converted by SCOT to acetoacetyl CoA which is then converted into acetyl CoA by MAT. Acetyl CoA enters the citric acid cycle and ATP is produced. SCOT activity is highest in cardiac muscle and the kidney, followed by the nervous system and skeletal muscle. ${ }^{7}$ Ketones can provide up to two-thirds of the energy requirements of the brain during periods of extended fasting or starvation. ${ }^{2}$

\section{Ketosis}

Normal circulating levels of ketone bodies is $<0.5 \mathrm{mM}$ and ketosis refers to elevated serum levels of ketones. ${ }^{2}$ This can either be hyperketonaemia or ketoacidosis. Hyperketonaemia is usually physiological due to prolonged fasting, intense exercise or a ketogenic (high-fat, low-carb) diet. Ketoacidosis is pathological and can be due to diabetes mellitus, cortisol deficiency, growth hormone deficiency, alcohol or salicylate poisoning and rare inborn errors of metabolism. ${ }^{2}$ The ketogenic diet (KD) has been used to treat refractory epilepsy ${ }^{8}$ and has found favour as a weight loss diet. The remaining part of this review will examine the low carbohydrate diet, the ketosis that it induces and the role that it can play in weight loss.

\section{The high-fat low-carbohydrate diet}

Low-carbohydrate (low-CHO) diets usually contain less than $100 \mathrm{~g}$ of $\mathrm{CHO}$ per day and consist of $50-60 \%$ calories from fat, $20-30 \%$ from protein and less than $20-30 \%$ from $\mathrm{CHO}{ }^{9}$ The most famous low-CHO diet is the 'Atkin's diet' which aims to reduce $\mathrm{CHO}$ intake to less than $50 \mathrm{~g} /$ day and ide-ally less than 20 g/day. ${ }^{10}$ In South Africa Tim Noakes published The Real Meal Revolution which advocated for a high-fat low-CHO diet based on the historical diet of William Banting, a 19th century British undertaker who was the first person known to popularise the low-CHO diet as a tool for weight loss. ${ }^{11}$ The so-called Banting diet has become popular especially for people who want to lose a large amount of weight rapidly but has led Professor Noakes to become involved in much controversy.

There are numerous versions of the low- $\mathrm{CHO}$ diet with various restrictions on the amount of $\mathrm{CHO}$ allowed per day. Thresholds of 100,50 and $20 \mathrm{~g}$ of $\mathrm{CHO}$ per day have all been advocated. However, the overall concept of these diets is to encourage the intake of animal fats and proteins while severely limiting or forbidding the intake of breads, grains, fruit and starchy vegetables. ${ }^{12}$ The end goal is to induce a state of ketosis, hence the term ketogenic diet (KD) and force the body to primarily use ketone bodies as a fuel source. The amount of $\mathrm{CHO}$ that will induce ketosis varies on an individual basis but is usually between 20 and $100 \mathrm{~g}$ of $\mathrm{CHO}$ per day.

It is important to note that the field of nutritional science is by no means exact and that the low-CHO KD is controversial amongst dietitians, nutritionists, physicians and the general public. The recommended fat intake and consumption of animal food products is much higher than conventional diet recommendations. Arguments in favour of the $\mathrm{KD}$ are that there are no actual minimum $\mathrm{CHO}$ requirements for humans, physiological ketosis is not harmful, insulin is not required for glucose utilisation, ketone bodies are able to regulate insulin and 
glucagon secretion and that a KD results in a significant reduction in fat mass with an increase in lean muscle mass in men. ${ }^{13}$ Opponents of the KD argue that the diet may lack sufficient micronutrients, can cause elevated uric acid levels, leads to dehydration and constipation and that the high saturated fat intake can increase the risk of heart disease. ${ }^{12}$

\section{Implications of the low-CHO diet}

\section{Glycogen availability}

The liver contains around $100 \mathrm{~g}$ of glycogen and this is depleted within 24 hours of $\mathrm{CHO}$ restriction. The remaining muscle glycogen $(400 \mathrm{~g})$ will be consumed in a few days to a week and at this stage a number of metabolic processes will occur. Gluconeogenesis from amino acids will ensure a normal blood glucose level, fat stores will be oxidised to provide energy for metabolic needs, and ketone bodies will be produced in the liver and used as a fuel source throughout the body. ${ }^{9,12}$

\section{Weight and blood glucose}

Significant decreases in fasting glucose have been observed in patients on a low-CHO diet, with reductions especially important in diabetic individuals and overweight subjects. This may be due to reduced insulin levels, ketone-induced reduced hepatic glucose output and weight loss itself, which affects insulin levels and sensitivity. ${ }^{12}$

The initial rapid weight loss observed in the KD is due to the depletion of glycogen stores and subsequent water loss associated with glycogen breakdown. Each gram of glycogen is stored with $3 \mathrm{~g}$ of water and hence the loss of around $500 \mathrm{~g}$ of glycogen can induce the loss of 1-2 kg of water. ${ }^{14}$ Further weight loss on the KD can be explained by a reduction in caloric intake, the appetite suppressing effects of $3 \mathrm{HB}$ and the satiating effects of protein and fat compared to $\mathrm{CHO} .{ }^{12}$ The reduced level of insulin on a KD also triggers the oxidation of triglycerides into fatty acids, which promotes the use of fatty acids as a fuel source, further promoting fat loss.

There is controversy as to the effect of a high-fat low-CHO diet on lean muscle mass and some authors argue that while fat loss is high on a high-fat diet, the loss of lean muscle mass is also high. However Volek et al. ${ }^{15}$ reported in a study on obese men who reduced their $\mathrm{CHO}$ intake over 12 weeks that the men had a significant reduction in fat mass $(-3.4 \mathrm{~kg})$ with a concomitant increase in lean body muscle $(+1.1 \mathrm{~kg})$. It has also been shown that as long as protein intake remains adequate the fat loss that occurs on a low-CHO diet is not accompanied by muscle loss. ${ }^{12}$

It is clear that consuming a low-CHO KD is associated with weight loss and specifically fat loss and that maintaining an adequate protein intake is important to prevent lean muscle loss as well as to improve satiety. Authors argue that this diet is more sustainable than the traditional low-fat diets usually recommended for weight loss and that a KD does not require calorie counting. ${ }^{11}$ This remains controversial with counter arguments that these diets are simply inducing weight loss because they are hypo-energetic and are not superior to other diets. ${ }^{12}$

\section{Lipid profiles}

Significant reductions in serum triglyceride levels have been consistently shown in individuals on a low- $\mathrm{CHO}$, high-fat diet and this reduction occurs regardless of the type or amount of fat they consume. This is due to removal of triglycerides from the blood as a fuel source. Equally important is the observed reduction in serum cholesterol levels observed even though cholesterol consumption is increased. ${ }^{12,13}$ Low-CHO diets increase high-density lipoprotein (HDL) cholesterol and decrease low-density lipoprotein (LDL) cholesterol. ${ }^{13}$ The reduction in LDL cholesterol is due to the reduced level of serum insulin and the presence of ketones which inhibit HMG-CoA reductase (the enzyme responsible for cholesterol synthesis). ${ }^{12}$ While the true relationship between triglyceride and $\mathrm{HDL} / \mathrm{LDL}$ cholesterol levels and heart disease is contentious, it is generally accepted that a lower serum triglyceride level, a higher HDL cholesterol and lower LDL cholesterol are all associated with lower risk of heart disease - these exact changes are consistently shown on a low-CHO, high-fat KD and the reductions in triglycerides and LDL cholesterol are greater than those seen with a low-fat diet. ${ }^{16}$

\section{Exercise performance}

It has been theorised that a low-CHO diet, by depleting muscle glycogen stores will impair physical performance during exercise due to a lack of immediately available energy for aerobic metabolism. However, intense exercise itself induces ketogenesis and ketones are easily used by skeletal muscle as a fuel source, while preserving glucose metabolism. ${ }^{12,13}$ Additionally, ketones provide more ATP gram for gram than $\mathrm{CHO}^{13}$ and a low-CHO KD also promotes the oxidation of fatty acids as a predominant muscle energy substrate. ${ }^{12}$ Exercise and starvation metabolism are paralleled in many ways and ketogenesis is an effective evolutionary mechanism that spares $\mathrm{CHO}$ reserves, prevents muscle breakdown and provides an alternate energy source. ${ }^{17}$ There does not appear to be any reduction in exercise performance in people consuming a low- $\mathrm{CHO}$ diet provided that protein intake is adequate.

\section{Long-term health implications}

Perhaps the greatest concern with the popularity of high-fat, low- $\mathrm{CHO}$ diets is the lack of clear evidence as to the long-term effects. This is due to a number of reasons: the amount of, and type of, fat (saturated vs polyunsaturated) varies substantially across these diets; the benefits of weight loss in general may obscure any negative consequences of the KD and the nature of nutritional studies is notoriously unreliable. It is not possible to randomise subjects to various diets for extended periods of time and then objectively measure health outcomes.

There appear to be no negative effects on cardiovascular risk with low-CHO diets in the short term and in fact the KD can 
reduce risk factors for heart disease as well as reduce levels of inflammatory markers that contribute to atherosclerosis. ${ }^{12}$ Longterm studies are required to determine the exact impact of the KD on cardiovascular health.

Some evidence from a small study suggests that the low$\mathrm{CHO}$ diet presents the kidney with an increased acid load and increases calcium loss in the urine and could impact on bone health as well as increase the risk of stone formation, but again more robust studies are required to determine the true impact of this. ${ }^{18}$

Finally, an area of concern with following a high-fat, low-CHO diet is the inevitable increase in the consumption of animal fats and meat that occur with such a diet and the potential risk of colorectal cancer that has been observed with diets high in meat. As with all aspects of dietary science, though this link is not clear and it is important to note that by reducing weight the risk of cancer is decreased, this could counterbalance any risks of increased meat consumption. ${ }^{12}$

\section{Conclusion}

Ketones are an important fuel source for the body in times of stress and yield more energy than $\mathrm{CHO}$. The use of a KD has been effective in treating refractory epilepsy and is associated with weight loss. The long-term effects of ketosis are not clear but an understanding of the physiology of ketogenesis and ketolysis may be able to explain the benefits (and risks) of following a KD.

\section{Conflict of interest}

The author declares no conflict of interest.

\section{Funding source}

None.

\section{ORCID}

T Leonard iD https://orcid.org/0000-0003-4426-3972

\section{References}

1. Power I, Kam P. Principles of physiology for the anaesthetist. 2nd ed. Boca Raton, FL: Taylor \& Francis Group; 2008.

2. Laffel L. Ketone bodies: a review of physiology, pathophysiology and application of monitoring to diabetes. Diabetes Metab Res Rev. 1999;15:412-26. https://doi. org/10.1002/(sici)1520-7560(199911/12)15:6<412::aid-dmrr72>3.0.co;2-8.

3. Partridge WM. Blood-brain barrier transport of glucose, free fatty acids, and ketone bodies. Adv Exp Med Biol. 1991;291:43-53. https://doi. org/10.1007/978-1-4684-5931-9_5.

4. McGarry J. Ellenberg \& Rifkin's diabetes mellitus. 5th ed. Porte D, Sherwin R, editors. New York: McGraw-Hill; 1996.

5. McGarry JD, Woeltje KF, Kuwajima M, Foster DW. Regulation of ketogenesis and the renaissance of carnitine palmitoyltransferase. Diabetes Metab Rev. 1989;5:271-84. https://doi.org/10.1002/dmr.5610050305.

6. Grabacka M, Pierzchalska M, Dean M, Reiss K. Regulation of ketone body metabolism and the role of PPARa. Int J Mol Sci. 2016;17:2093. https://doi. org/10.3390/ijms17122093.

7. Williamson D. Ketone body production and metabolism in the fetus and newborn. In: Polin R, Fox W, editors. Fetal and neonatal physiology. Philadelphia: WB Saunders Co; 1992.

8. Wheless JW. History of the ketogenic diet. Epilepsia. 2008;49:3-5. https://doi. org/10.1111/j.1528-1167.2008.01821.x.

9. Bilsborough SA, Crowe T. Low-carbohydrate diets: what are the potential short-and long-term health implications? Asia Pac J Clin Nutr. 2003;12:396-404.

10. Atkins R. Dr Atkin's new diet revolution. New York: Quill; 2002.

11. Noakes T, Proudfoot J, Creed S. The real meal revolution: The radical, sustainable approach to healthy eating. London: Robinson; 2015.

12. Adam-Perrot A, Clifton P, Brouns F. Low-carbohydrate diets: nutritional and physiological aspects. Obes Rev. 2006;7:49-58. https://doi. org/10.1111/j.1467-789X.2006.00222.x.

13. Manninen AH. Metabolic effects of the very-low-carbohydrate diets: misunderstood "villains" of metabolism. J Int Soc Sports Nutr. 2004;1:7-11.https:// doi.org/10.1186/1550-2783-1-2-7.

14. Bergstrom J, Furst $P$, Holmstrom BO, et al. Influence of injury and nutrition on muscle water and electrolytes: effect of elective operation. Ann Surg. 1981;193:810-6. https://doi.org/10.1097/00000658-198106000-00017.

15. Volek JS, Sharman MJ, Love DM, et al. Body composition and hormonal responses to a carbohydrate-restricted diet. Metab. 2002;51:864-70. https://doi. org/10.1053/meta.2002.32037.

16. Sharman MJ, Gomez AL, Kraemer WJ, Volek JS. Very low-carbohydrate and low-fat diets affect gasting lipids and post-prandial lipemia differently in overweight men. J Nutr. 2004;134:880-5. https://doi.org/10.1093/jn/134.4.880.

17. Cox PJ, Clarke K. Acute nutritional ketosis: implications for exercise performance and metabolism. Extreme Physiol Med. 2014;3:1-9. https://doi. org/10.1186/2046-7648-3-17.

18. Reddy ST, Wang C-Y, Sakhaee K, Brinkley L, Pak CYC. Effect of low-carbohydrate high-protein diets on acid-base balance, stone-forming propensity, and calcium metabolism. Am J Kidney Dis. 2002;40:265-74. https://doi.org/10.1053/ ajkd.2002.34504. 